\title{
Construction method of space-time code in MIMO-VLC system
}

\author{
Chengnan Sun ${ }^{1, *}$ \\ ${ }^{1}$ National Mobile Communications Research Laboratory,Southeast university,211189, Chengnan sun, \\ China
}

\begin{abstract}
In the recent years, the design of any order of space-time block code did not appear in the previous. In this paper, we investigate the criterion that how to design the space-time block code in Multiple input multiple output(MIMO) visible light communication(VLC) system. Based on the second order space-time block code, we give the design criterion of space-time block code of arbitrary order. Then we discuss the space-time block difference between traditional MIMO system and optical system. It is found that the space-time block codes of visible light system can be modified by the traditional space-time block codes and it can also realize independent decoding at the receiving end. In addition, in the receiver, how to realize fast calculation of the estimated value of the transmitted signal and a unified independent decoding scheme is presented.
\end{abstract}

\section{Introduction}

The space-time block code scheme in MIMO system was first proposed by Almouti in [1]. The full-rate space-time block code(STBC) is designed in the $2 \times 1$ MIMO system, and the full diversity gain can be obtained at the receiving end. The specific form is as follows

$$
\left[\begin{array}{cc}
x_{1} & x_{2} \\
-x_{2}^{*} & x_{1}^{*}
\end{array}\right]
$$

Where the $x_{i}$ is taken from a constellation point in the modulation mode and the $x_{i}^{*}$ means the conjugate of $x_{i}$. Since the signal sent in visible light communication system must be a non-negative real signal, it brings some difficulties to the design of space-time block code. In [2],the design scheme of space-time block code in MIMO-VLC system is proposed for the first time. Based on the On-Off-Keying(OOK) modulation and Pulse-PositionModulation(PPM) modulation, the traditional Almouti code is modified. The system can also obtain full set gain and the bit rate is 1 . The specific form is as follows

$$
\left[\begin{array}{ll}
x_{1} & x_{2} \\
\bar{x}_{2} & x_{1}
\end{array}\right]
$$

\footnotetext{
* Corresponding author: 220180748@seu.edu.cn
} 
The $\bar{x}_{2}$ mean $\mathrm{A}-x_{2}$, A is relate to the LED's light intensity. In [3], A space-time block code design scheme based on 2-PAM modulation is also proposed. In [4], the modulation method in the space-time block code design scheme of MIMO-VLC system is extended to M-PAM modulation with equal interval, and the design scheme of space-time block code with the number of transmitting antennas to the power of 2 is given. In these scenarios, the receiving end can be independently decoded using Maximum Likelihood (ML) criteria. In this paper, when the number of transmitting antennas is arbitrary, we propose a criterion to design the space-time code and in the receiver we propose a scheme to calculate the estimate of transmitting signal and the decoding scheme.

\section{Design Criteria of STBC Matrix}

\subsection{Transmitter design criteria}

In this section, we use the M-PAM which constellations are evenly spaced, the A is normalized to 1 , and the MIMO-VLC system is $\mathrm{N} \times 1$. There are $\mathrm{K}$ time slot. In every time slot, it has the same the channel state. the received signal is $\boldsymbol{y}$. the transmitted signal is $\boldsymbol{x}$, after going through the channel, the received signal's form is

$$
\left[\begin{array}{c}
y_{1} \\
\vdots \\
y_{m} \\
\vdots \\
y_{K}
\end{array}\right]=\left[\begin{array}{ccccc}
s_{11} & \cdots & s_{1 i} & \cdots & s_{1 N} \\
\vdots & \ddots & & & \vdots \\
s_{m 1} & & \ddots & & s_{m N} \\
\vdots & & & \ddots & \vdots \\
s_{K 1} & \cdots & s_{K i} & \cdots & s_{K N}
\end{array}\right] \times\left[\begin{array}{c}
h_{1} \\
\vdots \\
h_{m} \\
\vdots \\
h_{N}
\end{array}\right]
$$

where the $s_{j n}$ is the transmitted signal in the $j$ th time slot and at the $n$th antenna, $h_{j}$ is the $j$ th channel gain. $s_{j n} \in\left\{x_{i} \mid i=1,2, \cdots, n\right\} \bigcup\left\{\bar{x}_{i} \mid i=1,2, \cdots, n\right\}$.in this situation, at the receiving end, the distance measure if using ML detection is

$$
\begin{aligned}
& \sum_{j=1}^{K}\left|y_{j}-\sum_{n=1}^{N} s_{j n} h_{j n}\right|^{2}=\sum_{j=1}^{K}\left[y_{j}^{2}-2 y_{j} \sum_{n=1}^{N} s_{j n} h_{j n}+\left(\sum_{n=1}^{N} s_{j n} h_{j n}\right)^{2}\right] \\
& =\sum_{j=1}^{K}\left[y_{j}^{2}-2 y_{j} \sum_{n=1}^{N} s_{j n} h_{j n}+\left(\sum_{n=2}^{N} s_{j n} h_{j n}+s_{j 1} h_{j 1}\right)^{2}\right] \\
& =\sum_{j=1}^{K}\left[\begin{array}{l}
y_{j}^{2}-2 y_{j} \sum_{n=2}^{N} s_{j n} h_{j n}+\left(\sum_{n=2}^{N} s_{j n} h_{j n}\right)^{2} \\
\left.-2 y_{j} s_{j 1} h_{j 1}+2 s_{j 1} h_{j 1} \sum_{n=2}^{N} s_{j n} h_{j n}+\left(s_{j 1} h_{j 1}\right)^{2}\right]
\end{array}\right.
\end{aligned}
$$

In the formula (4), the first time slot's signal is separated from the entirety, so if the second time slot's signal is separated, it has the same form. Assuming that the $s_{m 1}=x_{1}, s_{m 2}=x_{2}$ in the $m$ th time slot. If you want to realize the independent decoding, the cross product term between the transmitted signals must be eliminated. Substitute $s_{m 1}=x_{1}, s_{m 2}=x_{2}$ into formula (4), it can produce two cross product terms between $x_{1}$ and 
$x_{2}$, this item is $2 x_{1} h_{m 1} x_{2} h_{m 2}$,inspired by [2], if in the lth time slot there is a $\bar{s}_{l 1}=\bar{x}_{1}$, $s_{l 2}=x_{2}$, because $\bar{x}_{1}=1-x_{1}$, in the lth time slot, the cross product terms is $2 x_{1} h_{l 1}\left(1-x_{2}\right) h_{l 2}$. In the case of the constant channel state, it can be eliminated and leave an offset variable $2 x_{1} h_{l 1} h_{l 2}$.In the same way, if $s_{m 1}=\bar{x}_{1}, s_{m 2}=\bar{x}_{2}, \bar{s}_{l 1}=\bar{x}_{2}, s_{l 2}=x_{1}$, it can have the same result. We can take the second-order minor from the STBC matrix as follow.

$$
\left[\begin{array}{cc}
x_{i} & x_{j} \\
\bar{x}_{j} & x_{i}
\end{array}\right],\left[\begin{array}{cc}
\bar{x}_{i} & \bar{x}_{j} \\
\bar{x}_{j} & x_{i}
\end{array}\right]
$$

In conclusion, for the STBC matrix of arbitrary transmitted antenna, assume the STBC matrix $\mathbf{A}$ is

$$
\mathbf{A}_{K \times N}=\left(\mathbf{A}_{1}, \mathbf{A}_{2}, \cdots, \mathbf{A}_{N}\right)
$$

If we taking any two columns from the matrix and put the two columns together into a matrix.

$$
\left[\mathbf{A}_{i}, \mathbf{A}_{j}\right]=\left[\begin{array}{cc}
a_{1 i} & a_{1 j} \\
\cdots & \cdots \\
a_{K i} & a_{K j}
\end{array}\right]
$$

when we take the arbitrary second order minor from the matrix in formula (6), if it can satisfy the formula (5) ( $x_{i}$ can represent any constellation points), and it is true for any case, the ML decision can independent decode at the receiving end. In addition, the number of time slot must be even because the transmitted signals' cross product item in two time slots of that form can be offset.

\subsection{Traditional STBC and STBC in VLC system}

There is a traditional STBC Matrix $\boldsymbol{B}$, assuming it can reach the maximum bit rate, $B$ is an orthogonal matrix. From the above derivation, if we do the same transformation like formula (7),

$$
\left[\mathbf{B}_{i}, \mathbf{B}_{j}\right]=\left[\begin{array}{cc}
b_{1 i} & b_{1 j} \\
\cdots & \cdots \\
b_{K i} & b_{K j}
\end{array}\right]
$$

if we take a second-order minor, it has two column vector. We can easily find that this two column vector's inner product is zero, which is true for any case. So the must have a offset $-b_{1 i} \times b_{1 j}$, if we change the $-b_{1 i} \triangleq \bar{b}_{1 i}$, we can find that can be use in VLC system.

\subsection{Receiver Design}

Assuming the VLC system's STBC satisfies the criterion, so the cross product can be eliminated. At the receiver, if we want to use the received signal and the channel information to construct the transmitted signal like the traditional Almouti code, we must 
calculate the estimate of transmitted signal. From the formula (4), if $s_{j 1} \triangleq \bar{s}_{j 1}$, we can derive that

$$
\begin{aligned}
& 2 y_{j} s_{j 1} h_{j 1}-2 s_{j 1} h_{j 1}^{2}+2\left(1-s_{j 1}\right) h_{j 1} \sum_{n=2}^{N_{t}} s_{j n} h_{j n}+h_{j 1}^{2} s_{j 1}^{2} \\
& =2\left(y_{j} h_{j 1}-h_{j 1}^{2}\right) s_{j 1}+2 h_{j 1} \sum_{n=2}^{N_{t}} s_{j n} h_{j n}+h_{j 1}^{2} s_{j 1}^{2}
\end{aligned}
$$

Notice that if we change $s_{j 1} \triangleq \bar{s}_{j 1}$, it brings a kind of offset like $h_{j 1}^{2}$ to the estimate of $s_{j 1}$,called $\hat{s}_{j 1}$ and brings a kind of offset like $\pm h_{i} h_{j}$ to every signal that transmitted in antenna. Next, we introduce how to calculate the $\pm h_{i} h_{j}$ and $h_{j}^{2}$. Defining the estimate of transmitted signal $\hat{x}_{i}=f\left(y_{1}, y_{2}, \cdots, y_{K}\right)+\Delta, \Delta$ is the offset. In a certain time slot, if we take two transmitted signal like $\left[\bar{x}_{j}, \cdots, x_{i}\right]$, and the other time slot have two transmitted signal $\left[x_{i}, \cdots, x_{j}\right]$ in the same channel. if we calculate the estimate of transmitted signal, you can find the offset $-h_{i} h_{j}$ is in the estimate in $x_{i}$. On the other hand, if we take two transmitted signal like $\left[\bar{x}_{j}, \cdots, \bar{x}_{i}\right]$, and the other time slot have two transmitted signal $\left[\bar{x}_{j}, \cdots, x_{i}\right]$ in the same channel. if we calculate the estimate of transmitted signal, you can find the offset $h_{i} h_{j}$ in the estimate of $x_{j}$. Then we calculate the left. We use the second order matrix for example.

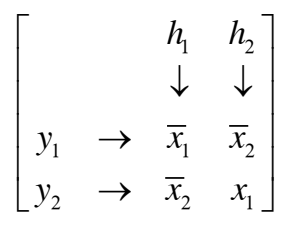

In this situation, determine the position of $x_{1}$, according to the position, the estimate of $x_{1}$ can be described as follow, if there is a $\bar{x}_{1}$,we can add negative number in the channel gain and a offset like $h_{j}^{2}$ in the correspond position.

$$
\begin{aligned}
& \hat{x}_{1}=f\left(y_{1}, y_{2}\right)+\Delta=-h_{1} y_{1}+h_{2} y_{2}+h_{1}^{2} \\
& \hat{x}_{2}=f\left(y_{1}, y_{2}\right)+\Delta=-h_{1} y_{2}-h_{2} y_{1}+h_{1}^{2}+h_{2}^{2}+h_{1} h_{2}
\end{aligned}
$$

If we design the STBC matrix in this criterion, it can easily calculate the estimate of transmitted signal and we can use the following formula to realize the ML detection.

$$
\underset{x_{i}}{\arg \min }\left(\hat{x}_{i}-x_{i}\right)^{2}+\left[\left(\sum_{t=1}^{N_{t}} h_{t}^{2}\right)-1\right] x_{i}^{2}
$$

\section{Simulation}

We use the criterion above to design a three transmitted antenna and four transmitted antenna. The channel is an exponential distribution that follows a parameter of 1 . In MIMO-VLC system, we conduct the Monte Carlo simulation. 


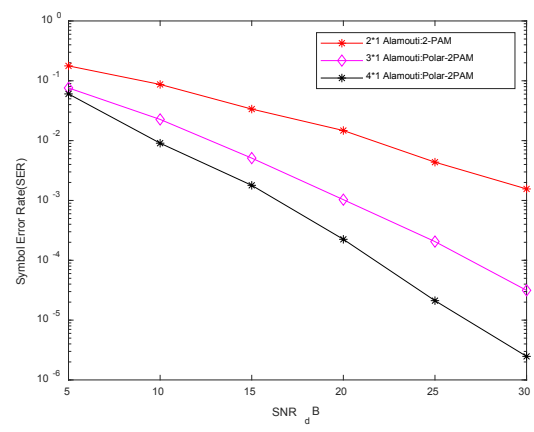

Fig. 1. Simulation of STBC in MIMO-VLC system.

It is shown in the simulation that if we use more transmitted antenna, we can get a better system performance and it also shown the design of the STBC in MIMO-VLC system is feasible.

This work was supported by the National Natural Science Foundation of China (NSFC) under grant 61871128 \& 61960206005 \& 61960206006 . This work was supported by the National Key Research and Development Program under grant 2018YFB1801905. This work was supported by Fundamental Research on Foreword Leading Technology of Jiangsu Province under grant BK20192002

\section{References}

1. S. M .Alamouti,"A simple transmit diversity technique for wireless communications," $\mathrm{i}$ nIEEE Journal on Selected Areas in Communications, vol. 16, no. 8, pp. 1451-1458, O ct. 1998, doi: 10.1109/49.730453.

2. Simon M K,Vilnrotter V A . Alamouti-type space-time coding for free-space optical co mmunication with direct detection[J]. IEEE Transactions on Wireless Communications, 2005, 4(1):35-39.

3. Safari M,Uysal M.Space-Time Coding versus Repetition Coding for Free-Space Optica 1 Communication[J]. Circuits, Systems and Computers, 1977. Conference Record. 197 7 11th Asilomar Conference on, 2007:1030-1033.

4. S. A. Naser and P. C. Sofotasios, "Generalization of Space-Time Block Coded-Spatial Modulation for High Data Rate VLC Systems (Invited Paper),"2020 3rd International Conference on Advanced Communication Technologies and Networking (CommNet), Marrakech, Morocco, 2020, pp. 1-5, doi: 10.1109/CommNet49926.2020.9199619. 